PROCEEDINGS OF THE

AMERICAN MATHEMATICAL SOCIETY

Volume 125, Number 3, March 1997, Pages 875-881

S 0002-9939(97)03746-5

\title{
FIXED POINT THEORY FOR COMPACT UPPER SEMI-CONTINUOUS OR LOWER SEMI-CONTINUOUS SET VALUED MAPS
}

\author{
DONAL O'REGAN
}

(Communicated by Palle E. T. Jorgensen)

\begin{abstract}
Fixed point theory is presented for compact u.s.c. and l.s.c. set valued maps.
\end{abstract}

\section{INTRODUCTION}

This paper presents new fixed point theory for compact upper semi-continuous (u.s.c.) and lower semi-continuous (l.s.c.) set valued mappings. Our results generalize some well known fixed point results for convex and nonconvex maps. Our paper is divided into two main sections. In section 2 we use a recent Leray-Schauder type alternative due to Ben-El-Mechaiekh and Idzik [3] for u.s.c., compact approachable maps with nonempty, compact values to establish a new fixed point result for such maps. Our theory generalizes some fixed point results in Ben-El-Mechaiekh and Deguire [2] and Granas [9]. In section 3 we establish via Michael's selection theorem [11] a nonlinear alternative of Leray-Schauder type for l.s.c., compact multifunctions with closed, convex values. Also a new fixed point result is given. This generalizes some results in Himmelberg, Porter and Van Vleck [10] and Tarafdar and Výborný [17]. A brief discussion of nonconvex maps is also given.

We now gather together some definitions and known facts. Let $X$ and $Y$ be topological spaces. A multifunction $f: X \rightarrow Y$ is a point to set function such that for each $x \in X, f(x)$ is a nonempty subset of $Y$. The function $f$ is u.s.c. if the set $f^{-1}(B)=\{x \in X: f(x) \cap B \neq \emptyset\}$ is closed for any closed set $B$ in $Y$ (equivalently, $f: X \rightarrow Y$ is u.s.c. if for any net $\left\{x_{\alpha}\right\}$ in $X$ and any closed set $B$ in $Y$ with $x_{\alpha} \rightarrow x_{0} \in X$ and $f\left(x_{\alpha}\right) \cap B \neq \emptyset$ for all $\alpha$ we have $\left.f\left(x_{0}\right) \cap B \neq \emptyset\right)$. The function $f: X \rightarrow Y$ is l.s.c. if the set $f^{-1}(A)$ is open for any open set $A$ in $Y . f: X \rightarrow Y$ is compact if $f(X)$ is relatively compact in $Y$. Finally the function $f$ is said to be point-compact if for each $x \in X, f(x)$ is a compact subset of $Y$. Now we state a result [16, proposition 1] which will be used frequently in this paper.

Theorem 1.1. Let $X$ and $Y$ be topological spaces and $f: X \rightarrow Y$ be a u.s.c., point-compact multifunction. Suppose $\left\{x_{\alpha}\right\}$ is a net in $X$ such that $x_{\alpha} \rightarrow x_{0}$. If $y_{\alpha} \in f\left(x_{\alpha}\right)$ for each $\alpha$, then there is a $y_{0} \in f\left(x_{0}\right)$ and a subnet $\left\{y_{\beta}\right\}$ of the net $\left\{y_{\alpha}\right\}$ such that $y_{\beta} \rightarrow y_{0}$.

Received by the editors October 16, 1995.

1991 Mathematics Subject Classification. Primary 47H10, 47H04. 
Theorem 1.1 immediately yields the following well known result.

Theorem 1.2. Let $X$ and $Y$ be topological spaces and $f: X \rightarrow Y$ be a u.s.c., point-compact multifunction. Suppose $\left\{x_{\alpha}\right\}$ is a net in $X$ and $y_{\alpha} \in f\left(x_{\alpha}\right)$ for each $\alpha$. If $x_{\alpha} \rightarrow x_{0}$ and $y_{\alpha} \rightarrow y_{0}$, then $y_{0} \in f\left(x_{0}\right)$.

\section{The UPPER SEMI-CONTINUOUS CASE}

We begin by describing the class of approachable set valued maps. Let $X$ and $Y$ be subsets of Hausdorff topological vector spaces $E_{1}$ and $E_{2}$ respectively, and let $F: X \rightarrow Y$ be a multifunction. Given two open neighborhoods $U$ and $V$ of the origins in $E_{1}$ and $E_{2}$ respectively, a $(U, V)$-continuous selection of $F$ is a continuous function $s: X \rightarrow Y$ satisfying

$$
s(x) \in(F[(x+U) \cap X]+V) \cap Y \text { for every } x \in X .
$$

$F$ is said to be approachable if it has a $(U, V)$-continuous selection for any open neighborhoods $\bar{U}$ and $V$ of the origins in $E_{1}$ and $E_{2}$ respectively. Let

$$
\mathcal{A}_{0}(X, Y)=\{F: X \rightarrow Y: F \text { is approachable }\}
$$

and

$$
\mathcal{A}(X, Y)=\left\{F \in \mathcal{A}_{0}(X, Y): F \text { is u.s.c. and compact valued }\right\} .
$$

Remark. For examples of approachable maps we refer the reader to $[2,3]$.

We now gather together three theorems from the literature which will be used in this section.

Theorem 2.1 ([2], pg.486, proposition 3.3). Let $X, Y$ and $Z$ be subsets of the Hausdorff topological vector spaces $E_{1}, E_{2}$ and $E_{3}$ respectively. Suppose $F: X \rightarrow$ $Y$ is a multivalued map with $F \in \mathcal{A}(X, Y)$ and $r: Z \rightarrow X$ is a continuous map (single valued). If $Z$ is compact then $\operatorname{Fr} \in \mathcal{A}(Z, Y)$.

Theorem 2.2 ([2], pg.496, corollary 7.3). Let $X$ be a convex subset of a locally convex Hausdorff linear topological space $E$ with $F \in \mathcal{A}(X, X)$ a compact map. Then $F$ has a fixed point (i.e. there exists $x \in X$ with $x \in F(x)$ ).

Theorem 2.3 ([3], pg.108, Theorem). Let $X$ be a closed subset of a locally convex Hausdorff linear topological space $E$ with $0 \in$ int $X$. In addition assume $F: X \rightarrow$ $E$ is a u.s.c., approachable, compact map with nonempty, closed values. Then either

(A1) $F$ has a fixed point in $X$; or

(A2) there exists $(\lambda, x) \in(0,1) \times \partial X$ such that $x \in \lambda F(x)$.

We now state and prove the main result of this section.

Theorem 2.4. Let $C$ be a complete convex subset of a metrizable (metric d) locally convex linear topological space $E$ with $Q$ a closed, convex, proper subset of $C$ and $0 \in Q$. Assume $F: Q \rightarrow C$ is a u.s.c., approachable, compact map with nonempty, compact values. In addition suppose

$$
\left\{\begin{array}{l}
\text { if }\left\{\left(x_{j}, \lambda_{j}\right)\right\}_{j=1}^{\infty} \text { is a sequence in } \partial Q \times[0,1] \text { converging to } \\
(x, \lambda) \text { with } x \in \lambda F(x) \text { and } 0 \leq \lambda<1 \\
\text { then there exists } j_{0} \in\{1,2, \ldots\} \text { with } \\
\left\{\lambda_{j} F\left(x_{j}\right)\right\} \subseteq Q \text { for each } j \geq j_{0} .
\end{array}\right.
$$


Finally assume either

$$
\left\{\begin{array}{l}
C \text { is compact and } U_{i}=\left\{x \in E: d(x, Q)<\frac{1}{i}\right\} \subseteq C \\
\text { for } i \text { sufficiently large, }
\end{array}\right.
$$

$\underline{\text { or }}$

$$
Q \subseteq \overline{c o}(\overline{F(Q)}) \text { and } U_{i} \subseteq \overline{c o}(\overline{F(Q)}) \text { for } i \text { sufficiently large, }
$$

$\underline{o r}$

$$
\left\{\begin{array}{l}
F r \in \mathcal{A}_{0}(C, C) \text { and } U_{i} \subseteq C \text { for } i \text { sufficiently large; here } \\
r: E \rightarrow Q \text { is a continuous retraction such that } \\
r(z) \in \partial Q \text { for } z \in E / Q
\end{array}\right.
$$

is satisfied. Then $F$ has a fixed point in $Q$.

Remarks. (i) The existence of a continuous retraction $r: E \rightarrow Q$ follows immediately from Dugundji's extension theorem [7, 18].

(ii) If $0 \in \operatorname{int} Q$ we may take

$$
r(x)=\frac{x}{\max \{1, \mu(x)\}}, x \in E .
$$

Here $\mu$ is the Minkowski functional [15] on $Q$, i.e. $\mu(x)=\inf \{\alpha>0: x \in$ $\alpha Q\}$. If $\operatorname{int} Q=\emptyset$ then $\partial Q=Q$. As a result we may (and do) choose the retraction $r$ above so that $r(z) \in \partial Q$ if $z \in E / Q$.

(iii) Notice (2.2a) and (2.2b) are only of interest if the space is normable.

(iv) If $F$ is convex valued then of course $F r$ is convex valued, so $F r$ is approachable [3], i.e. (2.2c) holds.

(v) Theorem 2.4 was proved by Furi and Pera [8], by a different method, when $F$ is single valued, compact and continuous. It was extended by O'Regan $[13,14]$ for the case when $F$ is single valued, condensing ( $P$-concentrative) and continuous.

Proof. Let $Z=C$ if $(2.2 \mathrm{a})$ or $(2.2 \mathrm{c})$ holds, and let $Z=\overline{c o}(\overline{F(Q)})$ if $(2.2 \mathrm{~b})$ is satisfied.

Remark. Note $\overline{c o}(\overline{F(Q)})$ is compact. To see this let $P$ be a defining system of seminorms. Fix $p \in P$. Since $\overline{F(Q)}$ is compact then $\overline{c o}(\overline{F(Q)})$ is precompact(totally bounded) [6] in the seminormed space $(E, p)$ for each $p \in P$. Thus $\overline{c o}(\overline{F(Q)})$ is precompact in $E$. Now since $\overline{c o}(\overline{F(Q)})$ is a closed precompact subset of $C$ and $C$ is complete, then $\overline{c o}(\overline{F(Q)})$ is compact [5].

Now let

$$
B=\{x \in Z: x \in \operatorname{Fr}(x)\} .
$$

We first show $B \neq \emptyset$. Notice $F r: Z \rightarrow Z$ is a u.s.c. (since $F$ is u.s.c. and $r$ is continuous), approachable (theorem 2.1 if $(2.2 \mathrm{a})$ or $(2.2 \mathrm{~b})$ occurs and by assumption if $(2.2 \mathrm{c})$ occurs $)$, compact map with nonempty, compact values. Theorem 2.2 implies that $F r$ has a fixed point, so $B \neq \emptyset$. We next claim that $B$ is closed. Let $\left(x_{\alpha}\right)$ be a net in $B$ with $x_{\alpha} \rightarrow x_{0} \in Z$. Theorem 1.2 implies $x_{0} \in \operatorname{Fr}\left(x_{0}\right)$, so $x_{0} \in B$. Thus $B$ is closed, and in fact $B$ is compact since $B \subseteq \operatorname{Fr}(B) \subseteq F(Q)$. 
It remains to show $B \cap Q \neq \emptyset$. To do this we argue by contradiction. Suppose $B \cap Q=\emptyset$. Then since $B$ is compact and $Q$ is closed there exists $\delta>0$ with $\operatorname{dist}(B, Q)>\delta$. Choose $m \in\{1,2, \ldots\}$ such that $1<\delta m$ and $U_{i} \subseteq Z$ for $i \in$ $\{m, m+1, \ldots\}$; here $U_{i}=\left\{x \in E: d(x, Q)<\frac{1}{i}\right\}$. Fix $i \in\{m, m+1, \ldots\}$. Since $\operatorname{dist}(B, Q)>\delta$, then $B \cap \overline{U_{i}}=\emptyset$. Now $0 \in U_{i}$ and $\mathrm{Fr}: \overline{U_{i}} \rightarrow C$ is a u.s.c., approachable, compact map with nonempty, compact values. Theorem 2.3 implies (since $\left.B \cap \overline{U_{i}}=\emptyset\right)$ that there exists $\left(y_{i}, \lambda_{i}\right) \in \partial U_{i} \times(0,1)$ with $y_{i} \in \lambda_{i} \operatorname{Fr}\left(y_{i}\right)$. Notice in particular since $y_{i} \in \partial U_{i}$ that

$$
\left\{\lambda_{i} \operatorname{Fr}\left(y_{i}\right)\right\} \nsubseteq Q \text { for each } i \in\{m, m+1, \ldots\} .
$$

Now consider

$$
D=\{x \in E: x \in \lambda F r(x) \text { for some } \lambda \in[0,1]\} .
$$

We first show $D$ is closed. To see this let $\left(x_{\alpha}\right)$ be a net in $D$ (i.e. for each $\alpha, x_{\alpha} \in \lambda_{\alpha} \operatorname{Fr}\left(x_{\alpha}\right)$ for some $\left.\lambda_{\alpha} \in[0,1]\right)$ with $x_{\alpha} \rightarrow x_{0} \in E$. Without loss of generality assume $\lambda_{\alpha} \rightarrow \lambda_{0} \in[0,1]$. We claim that

$$
N: E \times[0,1] \rightarrow C \text { given by } N(x, \lambda)=\lambda \operatorname{Fr}(x)
$$

is u.s.c. with nonempty, compact values. If the claim is true then theorem 1.2 implies $x_{0} \in N\left(x_{0}, \lambda_{0}\right)$, i.e. $x_{0} \in \lambda_{0} \operatorname{Fr}\left(x_{0}\right)$, so $D$ is closed. To see that $N$ is u.s.c. let $\Omega$ be a closed subset of $C,\left(y_{\alpha}, t_{\alpha}\right)$ a net in $E \times[0,1],\left(y_{\alpha}, t_{\alpha}\right) \rightarrow\left(y_{0}, t_{0}\right)$ and $t_{\alpha} \operatorname{Fr}\left(y_{\alpha}\right) \cap \Omega \neq \emptyset$. We must show $t_{0} \operatorname{Fr}\left(y_{0}\right) \cap \Omega \neq \emptyset$. Suppose $w_{\alpha} \in \operatorname{Fr}\left(y_{\alpha}\right)$ with $t_{\alpha} w_{\alpha} \in \Omega$. Since $F r$ is u.s.c., there exist (theorem 1.1) $w_{0} \in F r\left(y_{0}\right)$ and a subnet $\left(w_{\beta}\right)$ of $\left(w_{\alpha}\right)$ with $w_{\beta} \rightarrow w_{0}$. Since $\Omega$ is closed we have $t_{0} w_{0} \in \Omega$. Consequently $t_{0} \operatorname{Fr}\left(y_{0}\right) \cap \Omega \neq \emptyset$, so $N: E \times[0,1] \rightarrow C$ is u.s.c. Thus $D$ is closed and also compact since $D \subseteq c o(F(Q) \cup\{0\})$. This together with $d\left(y_{j}, Q\right)=\frac{1}{j},\left|\lambda_{j}\right| \leq 1$ (for $j \in\{m, m+1, \ldots\}$ ), implies that we may assume without loss of generality that $\lambda_{j} \rightarrow \lambda^{\star}$ and $y_{j} \rightarrow y^{\star} \in \partial Q$. Also, since $y_{j} \in \lambda_{j} \operatorname{Fr}\left(y_{j}\right)$, we have from theorem 1.2 that $y^{\star} \in \lambda^{\star} \operatorname{Fr}\left(y^{\star}\right)$ (note from above that $N: \overline{U_{m}} \times[0,1] \rightarrow C$ given by $N(u, \lambda)=\lambda \operatorname{Fr}(u)$ is u.s.c. with nonempty, compact values). If $\lambda^{\star}=1$, then $y^{\star} \in \operatorname{Fr}\left(y^{\star}\right)$, which contradicts $B \cap Q=\emptyset$. Hence we may assume $0 \leq \lambda^{\star}<1$. But in this case, (2.1) with $x_{j}=r\left(y_{j}\right) \in \partial Q, x=y^{\star}=r\left(y^{\star}\right)$ implies that there exists $j_{0} \in\{1,2, \ldots\}$ with $\left\{\lambda_{j} \operatorname{Fr}\left(y_{j}\right)\right\} \subseteq Q$ for each $j \geq j_{0}$. This contradicts (2.3). Thus $B \cap Q \neq \emptyset$, so there exists $x \in Q$ with $x \in F r(x)$, i.e. $x \in F(x)$.

Let $X$ and $Y$ be subsets of a locally convex Hausdorff linear topological space E. More generally, let

$$
\mathcal{B}_{0}(X, Y)=\{F: X \rightarrow Y: F \text { satisfies property } B\}
$$

and let $\mathcal{B}(X, Y)$ be the set of maps $F: X \rightarrow Y$ which are u.s.c., compact with nonempty, compact values and satisfy a property $B$ (which we will not specify). Suppose the following properties are satisfied:

if $X$ is convex and $F \in \mathcal{B}(X, Y)$ then $F$ has a fixed point in $X$

and

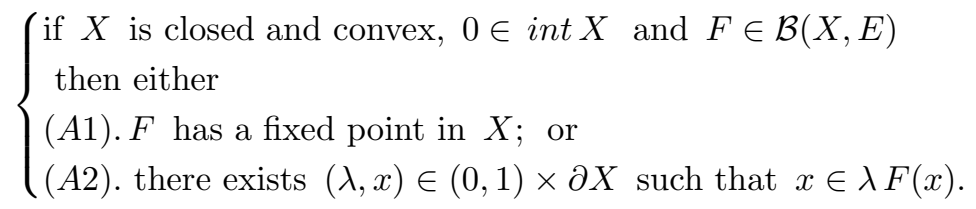


With the above properties, essentially the same reasoning as in theorem 2.4 establishes the following result.

Theorem 2.5. Let $C$ be a complete convex subset of a metrizable (metric d) locally convex linear topological space $E$ with $Q$ a closed, convex, proper subset of $C, 0 \in Q$ and $U_{i}=\left\{x \in E: d(x, Q)<\frac{1}{i}\right\} \subseteq C$ for $i$ sufficiently large. Assume $F \in \mathcal{B}(Q, C),(2.1)$ holds and also that

$$
\left\{\begin{array}{l}
F r \in \mathcal{B}_{0}(C, C) ; \text { here } r: E \rightarrow Q \text { is a continuous retraction } \\
\text { such that } r(z) \in \partial Q \text { for } z \in E / Q
\end{array}\right.
$$

is satisfied. Then $F$ has a fixed point in $Q$.

Remark. For examples of classes of maps $\mathcal{B}_{0}(X, Y)$ we refer the reader to [9]. For example, property $B$ could be that the values of the maps are $R_{\delta}$ sets.

\section{The LOWER SEMI-CONTINUOUS CASE}

We begin by establishing a nonlinear alternative of Leray-Schauder type for l.s.c. set valued maps.

Theorem 3.1. Let $Q$ be either (i) a closed convex subset of a Fréchet space (complete metrizable locally convex linear topological space) E, or (ii) a complete, convex, compact, metrizable subset of a locally convex Hausdorff linear topological space $E$. Assume $U$ is a relatively open subset of $Q, 0 \in U$ and $U$ is convex. Suppose $F: \bar{U} \rightarrow Q$ is a l.s.c., compact map with closed, convex values. Then either

(A1) $F$ has a fixed point in $\bar{U}$; or

(A2) there exist $u \in \partial U$ and $\lambda \in(0,1)$ with $u \in \lambda F(u)$.

Remark. $\bar{U}$ and $\partial U$ denote the closure and boundary of $U$ in $Q$ respectively.

Proof. Define the retraction $r: Q \rightarrow \bar{U}$ by

$$
r(x)=\frac{x}{\max \{1, \mu(x)\}}, x \in Q,
$$

where $\mu$ is the Minkowski functional [15] on $\bar{U}$, i.e. $\mu(x)=\inf \{\alpha>0: x \in \alpha \bar{U}\}$. Consider the mapping $F r: Q \rightarrow Q$. Notice $F r$ is an l.s.c., compact map with closed, convex values. Michael's selection theorem [11, theorem 1.2] implies that there exists a continuous function $f: Q \rightarrow Q$ such that $f(x) \in \operatorname{Fr}(x)$ for all $x \in Q$. Next Tychonoff's theorem [7] implies that $f$ has a fixed point, which is also of course a fixed point of $F r$, i.e. there exists $y \in Q$ with $y \in \operatorname{Fr}(y)$. Thus if $z=r(y) \in \bar{U}$ we have $z \in r F(z)$, i.e. $z=r(w)$ for some $w \in F(z)$, i.e. $z \in \bar{U}$ is a fixed point of $r F$. Now either $w \in \bar{U}$ or not. If $w \in \bar{U}$ then $r(w)=w$, so $(A 1)$ occurs. If $w \notin \bar{U}$ then $z=r(w)=\frac{w}{\mu(w)}$, so $z \in \lambda F(z)$ where $\lambda=\frac{1}{\mu(w)}<1$, i.e. $(A 2)$ occurs.

Remark. In theorem 3.1 conditions were put on $Q, E$ and $F$ so that Michael's selection theorem could be applied. Of course results could be established if we used other selection theorems for l.s.c. set valued maps (convex and nonconvex); see $[1,4,12]$ for example. This remark also applies to the next theorem.

We now prove a new fixed point result for l.s.c., compact set valued maps. 
Theorem 3.2. Let $C$ be either (i) a closed convex subset of a Fréchet space (metric d) $E$ or (ii) a complete, convex, compact subset of a metrizable (metric d) locally convex linear topological space $E$. Assume $Q$ is a closed, convex, proper subset of $C$ with $0 \in Q$ and $U_{i}=\left\{x \in E: d(x, Q)<\frac{1}{i}\right\} \subseteq C$ for $i$ sufficiently large. Suppose $F: Q \rightarrow C$ is an l.s.c., compact map with closed, convex values, and that (2.1) holds. Then $F$ has a fixed point.

Proof. Let $r: E \rightarrow Q$ be a continuous retraction with $r(z) \in \partial Q$ for $z \in E / Q$. By Michael's selection theorem [11] (since $F r: C \rightarrow C$ is an l.s.c., compact map with closed, convex values) there exists a continuous function $f: C \rightarrow C$ such that $f(x) \in \operatorname{Fr}(x)$ for all $x \in C$. Consider

$$
B=\{x \in C: x=f(x)\} .
$$

Now $B \neq \emptyset$ by Tychonoff's theorem. Also $B$ is clearly closed and compact, since $B \subseteq f(B) \subseteq F(Q)$. It remains to show $B \cap Q \neq \emptyset$. Suppose $B \cap Q=\emptyset$. Then there exists $\delta>0$ with $\operatorname{dist}(B, Q)>\delta$. Choose $m \in\{1,2, \ldots\}$ such that $1<\delta m$ and $U_{i} \subseteq C$ for $i \in\{m, m+1, \ldots\}$. Fix $i \in\{m, m+1, \ldots\}$. Since $\operatorname{dist}(B, Q)>\delta$, then $B \cap \overline{U_{i}}=\emptyset$. Now $f: \overline{U_{i}} \rightarrow C$ is a continuous, compact map, so by the nonlinear alternative for single valued maps [7] there exists (since $\left.B \cap \overline{U_{i}}=\emptyset\right)\left(y_{i}, \lambda_{i}\right) \in \partial U_{i} \times(0,1)$ with $y_{i}=\lambda_{i} f\left(y_{i}\right)$. Now since $y_{i} \in \partial U_{i}$ we have $\lambda_{i} f\left(y_{i}\right) \notin Q$ for $i \in\{m, m+1, \ldots\}$, and so

$$
\left\{\lambda_{i} \operatorname{Fr}\left(y_{i}\right)\right\} \not \subset Q \text { for each } i \in\{m, m+1, \ldots\} .
$$

Let

$$
D=\{x \in C: x=\lambda f(x) \text { for some } \lambda \in[0,1]\} .
$$

Since $D$ is compact and $d\left(y_{j}, Q\right)=\frac{1}{j},\left|\lambda_{j}\right| \leq 1$ (for $j \in\{m, m+1, \ldots\}$ ), we may assume without loss of generality that $\lambda_{j} \rightarrow \lambda^{\star}$ and $y_{j} \rightarrow y^{\star} \in \partial Q$. Also, since $y_{j}=\lambda_{j} f\left(y_{j}\right)$ we have $y^{\star}=\lambda^{\star} f\left(y^{\star}\right)$. Now $\lambda^{\star} \neq 1$ since $B \cap Q=\emptyset$. Thus $0 \leq$ $\lambda^{\star}<1$ and $y^{\star} \in \lambda^{\star} \operatorname{Fr}\left(y^{\star}\right)$. But in this case (2.1) with $x_{j}=r\left(y_{j}\right), x=y^{\star}=r\left(y^{\star}\right)$ implies that there exists $j_{0} \in\{1,2, \ldots\}$ with $\left\{\lambda_{j} \operatorname{Fr}\left(y_{j}\right)\right\} \subseteq Q$ for each $j \geq j_{0}$. This contradicts (3.1), so $B \cap Q \neq \emptyset$.

Remark. One could use the ideas in this section together with ideas in [14] to obtain new results of Leray-Schauder and Furi-Pera type for l.s.c., condensing $(P-$ concentrative) maps.

\section{REFERENCES}

[1] H. Ben-El-Mechaiekh, Fixed points for compact set-valued maps, Q. and A. in General Topology, 10(1992), 153-156. CMP 92:07

[2] H. Ben-El-Mechaiekh and P. Deguire, Approachability and fixed points for non-convex setvalued maps, Jour. Math. Anal. Appl., 170(1992), 477-500. MR 94a:54103

[3] H. Ben-El-Mechaiekh and A.Idzik, A Leray-Schauder type theorem for approximable maps, Proc. Amer. Math. Soc., 122(1994), 105-109. MR 94k:54074

[4] H. Ben-El-Mechaiekh and M.Oudadess, Some selection theorems without convexity, Jour. Math. Anal. Appl., 195(1995), 614-618. CMP 96:02

[5] R. Cristescu, Topological vector spaces, Noordhoff Int. Publ., Leyden, 1977. MR 56:12802

[6] J. Daneš, Generalized concentrative mappings and their fixed points, Comment. Math. Univ. Carolinae, 11(1970), 115-136. MR 41:7668

[7] J. Dugundji and A.Granas, Fixed point theory, Monografie Matematyczne, PWN, Warsaw, 1982. MR 83j:54038 
[8] M. Furi and P. Pera, A continuation method on locally convex spaces and applications to ordinary differential equations on noncompact intervals, Ann. Polon. Math., 47(1987), 331346. MR 89d: 47122

[9] A. Granas, On the Leray-Schauder alternative, Top. Methods in Nonlinear Anal., 2(1993), 225-231. MR 94j:47082

[10] C. J. Himmelberg, J. R. Porter and F. S. van Vlech, Fixed point theorems for condensing multifunctions, Proc. Amer. Math. Soc., 23(1969), 635-641. MR 39:7480

[11] E. Michael, A selection theorem, Proc. Amer. Math. Soc., 17(1966), 1404-1406. MR 34:3551

[12] E. Michael and C. Pixley, A unified theorem on continuous selections, Pac. Jour. of Maths., 87(1980), 187-188. MR 81m:54025a

[13] D. O'Regan, Fixed point theory for the sum of two operators, Applied Mathematics Letters, $\mathbf{9}(1996), 1-8$.

[14] D. O'Regan, Some fixed point theorems for concentrative mappings between locally convex linear topological spaces, Nonlinear Anal., to appear.

[15] A. J. B. Potter, An elementary version of the Leray-Schauder theorem, Jour. London Math. Soc., 5(1972), 414-416. MR 47:904

[16] C. H. Su and V. M. Sehgal, Some fixed point theorems for condensing multifunctions in locally convex spaces, Proc. Amer. Math. Soc., 50(1975), 150-154. MR 52:1430

[17] E. Tarafdar and R. Výborný, Fixed point theorems for condensing multivalued mappings on a locally convex topological space, Bull. Austral. Math. Soc., 12(1975), 161-170. MR 52: 4048

[18] E. Zeidler, Nonlinear functional analysis and its applications, Vol I, Springer Verlag, New York, 1986 MR 87f:47083

Department Of Mathematics, University College Galway, Galway, Ireland

E-mail address: Donal.ORegan@UCG.IE 